\title{
Anti-protozoan Effects of Methanol Extracts of the Ferula szowitsiana on the Trichomonas Vaginalis Trophozoites in vitro
}

\author{
Majid Khanmohammadi ${ }^{1 *}$, Shalaleh Ganji ${ }^{2}$, Siamak Reyhani $\operatorname{Rad}^{1}$
}

\begin{abstract}
Objectives: Trichomonas vaginalis which is flagellated unicellular organism is the causative agent of vaginitis. Medications used to treat trichomoniasis often have unpleasant side effects or are not effective. Given the prevalence of this disease, the production of safe, effective and affordable medicines is necessary. Various species of ferula are antibacterial and anti-fungal and anti- protozoan. So far, no study has been done on the effect of Ferula szowitsiana on trophozoite of Trichomonas vaginalis.

Materials and Methods: Coma (Ferula), native to Eastern Azerbaijan province, was collected and methanol extracts were prepared. Trophozoite of Trichomonas vaginalis was cultured in vitro in CPLM medium and the effect of the mentioned extracts on the growth and survival of Trichomonas vaginalis trophozoite was measured by Mack-master slide.

Results: Concentrations of 2 and $3 \mathrm{mg} / \mathrm{ml}$ in Methanol extracts of $F$. szowitsiana and could inhibit the growth of Trichomonas vaginalis trophozoite completely after 72 -hour incubation. Trichomonas vaginalis trophozoite survived at concentrations of 0.5 and 0.375 and $0.25 \mathrm{mg} / \mathrm{ml}$ and methanol extract of $F$. szowitsiana were 42,52 and $68 \%$, respectively. The reduction of the number of parasites was visible 48 hours after incubation which was statistically significant $(\mathrm{P}<0.05)$.

Conclusion: Ferula extract in different concentrations inhibited the growth of the dose-dependent trophozoite of Trichomonas vaginalis. The results of this study showed that native species of $F$. szowitsiana is suitable for the study of anti-trichomonas nature of it in vitro. Therefore, further study and identification of effective components in the methanol extract in the studied species are required.
\end{abstract}

Keywords: Coma (Ferula), Trichomonas vaginalis, Trophozoite

\section{Introduction}

Trichomonas vaginalis was first discovered and named by Donne in 1836 (1). This protozoan causes vaginitis in women and rarely urethritis and sometimes prostatitis in men. The symptoms either do not or rarely appear in men and starts with Itching and discharge in women (1). About 180 million new infections are reported in all the over world each year. This protozoan is one the most common causes of non-viral sexually transmitted diseases (1). Given the importance of the disease and the problems of controlling and combating due to sexual transmission, the drug resistance and the lack of appropriate and certain vaccines, a need is felt for appropriate, safe, inexpensive and accessible medical approaches. However, toxicity and side effects of chemical drugs, the cost and difficulty of producing pharmaceutical chemicals have the scientists pay attention to herbal drugs that have fewer side effects and are economically more affordable, while studying various chemical treatments. On the other hand herbal medicine has been known to humans since ancient times and the history of human knowledge of plants and their use coincided with the creation of man. The archaeological evidence estimated that it dates back at least five thousand years BC and some valuable texts have been recorded in this regard in the valuable works of Iranian scientists (2). On the other hand, due to the new approach of science to herbs, natural substances instead of synthetic chemicals and back towards traditional medicine gets more than 80 percent of the world pharmaceutical research centers to focus on the use of herbal materials. Today, the number of formal herbal medicines used in the treatment of diseases compared to the total number of official drug in the world is increasing; the highest value of this proportion is allocated to countries such as China and India, with about $70 \%$, and the lowest to countries like America, with about $20 \%$ (3). Unfortunately the ratio is very low in Iran, making up less than $1 \%$ in years 1991-2001, and is currently around 5\%. Natural products from medicinal plants provide a large source of drugs and are the foundation for the development of drug compounds. Herbal medicines have greater public acceptance and generally have fewer side effects and are used more than chemical medicines

Received 2 April 2014, Revised 27 April 2014, Accepted 14 May 2014, Available online 20 July 2014

${ }^{1}$ Department of Laboratory Sciences, Marand Branch, Islamic Azad University, Marand, Iran. ${ }^{2}$ Department of Pediatric, Children's Hospital, Tabriz University of Medical Sciences, Tabriz, Iran.

* Corresponding Author: Majid Khanmohammadi, Department of Laboratory Sciences, Marand Branch, Islamic Azad University, Marand, Iran. Tel: +989141165049, Email: mkh593@marandiau.ac.ir 
in the treatment of chronic diseases (3). Considering the emphasis of different schools of alternative medicine, and in particular public medicine on the use of medicinal plants in the treatment of trichomoniasis, the most comprehensive studies on plants in different regions of the world have been done in recent years and its importance in terms of health should be considered. A major trend has arisen for the use of medicinal plants to treat a variety of diseases in the world so that almost 20,000 species of plants are used for medical purposes. Most published studies have not made the effectiveness of the mechanisms of essences from medicinal plants clear. However, some have raised the hypothesis that essences may be involved in the biochemical directions (4). Ferula is a genus of about 170 species of flowering plants in the family Apiaceae, These species are mainly in Middle east, mostly growing in arid climates. They are herbaceous perennial plants growing to 1-4 m tall, with stout, hollow, somewhat succulent stems. The leaves are Tripinnate or even more finely divided, with a stout basal sheath clasping the stem. The flowers are yellow, produced in large umbels (5). Various species of ferula are of the recommended plants for this purpose whose anti-bacterial, anti-fungal and anti-leishmanial nature have been proven (6). This genus of ferula is as a source of biologically active compounds such as derivatives of Sesquiterpene (7-10) and the Coumarin (11-15), sulfur compounds $(16,17)$ and Coumarin glycosides (18). The conducted studies on the compounds and biological materials obtained from plants have identified the effects including inhibition of microbial pigment formation (19), anti-virus (20), antimycobacterium (21), induction of apoptosis of Melanoma cancerous cells (22), inhibitor of matrix metalloproteinase and cancer-preventive and anti-leishmaniasis (14). Devising new, affordable, accessible and safe treatments are among the result of this project which can provide valuable services to the social health in terms of treatment, control and prevention of trichomoniasis. Based on the carried out studies the anti-bacterial and anti-fungal, antiprotozoan nature of the biological compounds found in this plant has been proven, but no study has been done on the effect of the extracts of $F$. szowitsiana and F. rigidula on trophozoite of Trichomonas vaginalis. Therefore, the present study aimed to examine the anti-protozoan effect of methanol extracts in these plants on Trichomonas vaginalis trophozoite in vitro.

\section{Material and Methods}

The roots of the two species of F. szowitsiana were collected in Khoja, the suburb of Tabriz, around the end of the growing season. The plants were dried in the shade and then the stems and roots of the dried plants were powdered. Methanol extract was provided using methanol solvent at room temperature through the Soxhlet method. Therefore, for every 100 grams of plant, $500 \mathrm{ml}$ of methanol was added and the mixture was placed on shaker every few hours for 15-30 minutes to let the effective constituent move out and after 48 hours, it has been smoothed twice using Whatman filter paper. Extracts prepared at temperatures of $40-50{ }^{\circ} \mathrm{C}$ were dried using vacuum distillation.

\section{Cultivation of Trichomonas vaginalis trophozoite}

For the mass cultivation, in sterile conditions, we cultured the parasite samples CPLM (Hi Media Laboratories PVT Ltd Company) in temperature of $37^{\circ} \mathrm{C}$. Then, we have added $5 \mathrm{ml}$ of the medium to the CPLM culture medium and have put at a temperature of $24-22^{\circ} \mathrm{C}$. After 72 hours, we review the medium in terms of parasite growth and the absence of bacterial and fungal contamination and again we made medium size 5 times bigger and we repeated this every 72 hours until the intended parasite volume was obtained. All tubes were examined unknowingly in terms of viability and motility from the beginning of cultivation with 15- minute intervals up to 2 hours and then in $3,4,5$, $6,12,24,48,72$ hours (18).

The effect of different concentrations of Methanol extracts (F. szowitsiana) on Trophozoite of Trichomonas vaginalis This study was an observational-experimental study. From the trophozoite of Trichomonas vaginalis in vitro in stationary phase, $100 \mathrm{ml}$ was removed and added into a 96-hole well plate was added as triplicate so that $5 \times 105$ promastigotes was put in each well. Then different extracts and drug control were prepared from the 3, 2, 1 , and 0.5 and 0.375 and $0.25 \mathrm{mg} / \mathrm{ml}$ concentrations respectively and $10 \mathrm{ml}$ was added to the 96 -hole well plate as triplicate. Metronidazole, and Amphotericin B were used as a positive control and Dimethyl sulfoxide, DMSO (Merk, Germany) was used as a negative control. The plate incubated at $24{ }^{\circ} \mathrm{C}$ and $5 \%$ - pressure of CO2 for 72 hours (18). After 72 hour incubation, the survival of trophozoite was reviewed with Mack-master slide. The experiments were repeated three times separately. In this experimental study, the statistical analysis was done using one way ANOVA and Post-hoc Dunnett's test $(\mathrm{P}<0.05)$ to compare the means of the different case groups and control group.

\section{Results}

In the review of the effect of ferula methanol extracts on Trichomonas vaginalis compared with the control groups, it was identified that methanol extracts of all the different concentrations of F. szowitsiana inhibited the growth and multiplication of Trichomonas vaginalis trophozoite in a dose-dependent manner. These effects were statistically significant $(\mathrm{P}<0.05)$. Concentrations of 2 and $3 \mathrm{mg} /$ $\mathrm{ml}$ of methanol extract of $F$. szowitsiana after 72-hour incubation inhibited the growth of Trichomonas vaginalis trophozoite completely. Trichomonas vaginalis trophozoite survived at concentrations of 0.5 and 0.375 and $0.25 \mathrm{mg} /$ $\mathrm{ml}$ and methanol extract of $F$. szowitsiana were 42,52 and $68 \%$ respectively and at concentrations of 0.5 and 0.375 and $0.25 \mathrm{mg} / \mathrm{ml}$ respectively (Figure 1). The reduction of the number of parasites was also observed 48 hours after incubation which was statistically significant $(\mathrm{P}<0.05)$. Also in the control group, without Methanol extract of 


\section{Concentration of metanol extract (F. szwitsiana)}

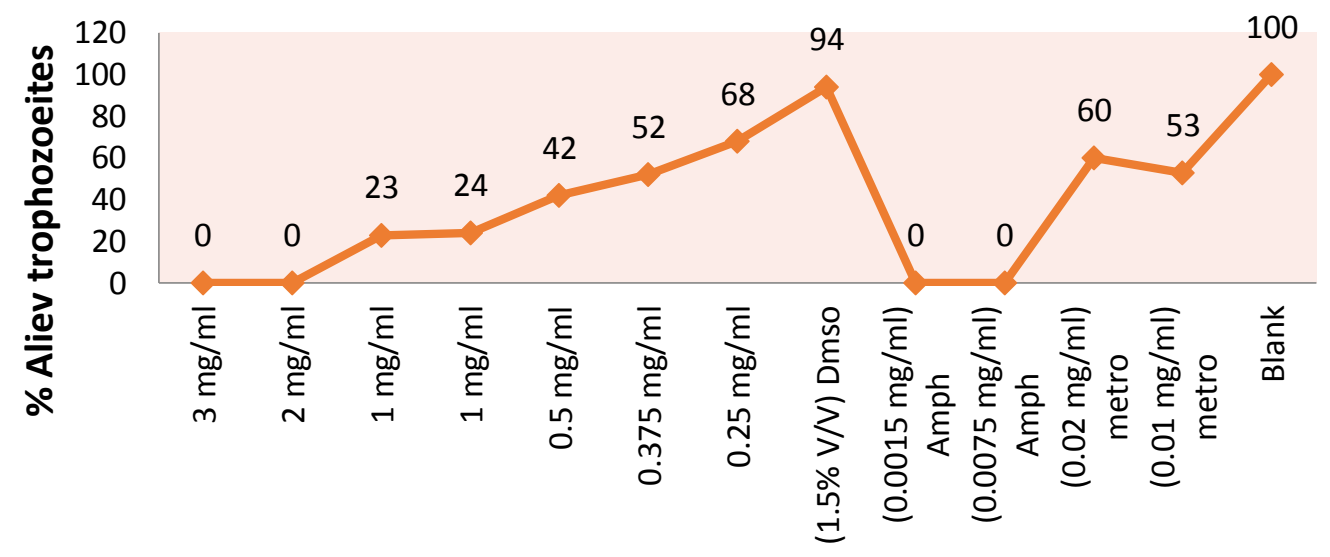

Figure 1. Percentage of live trophozoite dependent on different doses of Methanol extract of $F$. szowitsiana, Amphotericin B, Metronidazole and DMSO, reviewing with Mack-master slide.

F. szowitsiana an increase was found in the number of parasites that was significant. Amphotericin B at the concentrations of 0.0015 and $0.0075 \mathrm{mg} / \mathrm{ml}$ inhibited the parasite growth completely. After adding Metronidazole to the culture medium treated with extracts of F. szowitsiana, concentrations of 0.01 and $0.02 \mathrm{mg} / \mathrm{ml}, 53$ and $60 \%$ of trophozoite survived respectively. LD50 (Concentration that killed half of Trichomonas vaginalis trophozoite) was obtained for Methanol extracts of F. szowitsiana with dose of $0.360 \mathrm{mg} / \mathrm{ml}$, respectively (Figure 1).

\section{Discussion}

Trichomoniasis is the most common sexually transmitted disease worldwide caused by Trichomonas vaginalis (1). The parasite is responsible for $11 \%$ of all non-gonorrhea urethritis, prostatitis, epididymitis cases as well as infertility in men $(23,24)$. The common drug for treatment of this disease is Metronidazole. However, numerous reports from many countries regarding the prevalence of resistance to the drug and its carcinogenic and teratogenic complications (especially in the first trimester) had the researchers search for alternative drugs with fewer side effects (25). Medicinal herbs have been used worldwide, both in developing and developed countries since ancient times. It can be widely used for a variety of reasons such as the advantage of minimal side effects, improved better acceptance of patient due to the recommendation of alternative medicine and the use of past generations, or the reasonable price of herbal medicine. The therapeutic effects of Medicinal herbs are in consistent with normal physiological functioning of the human body. The most important species used in past medicine in Iran include F. gumosa and F. assa-foetida, the latter produces Oleo gum resin which has various medicinal uses in traditional medicine. Oleo gum resin can also be obtained from other species of ferula such as F. rigidula, F. foetida, F. alliacea. Resin F. gumosa known as Barijeh has been used for a long time. However, the most important secondary metabolites in plants from ferula species are Sesquiterpene derivatives. Since major part secondary metabolites in all species ferula are stored in roots, the plant root was used in this study $(3,5)$. In the present study, the number of live parasites in vitro dependent on methanol extract of the plant, significantly reduced. The antimicrobial effects of Ferula have been studied by various researchers, using different methods. In the studies conducted on the effects of ferula, the major microorganisms that have been studied included: Staphylococcus aureus, Escherichia coli and Leishmania major (14,16-18). In 2007, Shahverdi et al. showed that Galbanic acid in the roots of F. szowitsiana increased the antibacterial activity of penicillin $G$ and Cephalexin against Staphylococcus aureus (16). Also, in 2009, the study of Fazlibazaz et al. demonstrated the effect of Galbanic acid in the roots of F. szowitsiana as a modulator of multi-drug resistance in clinical isolates of Staphylococcus aureus and Escherichia coli (17). Fazlibazaz and Colleagues in another study declared that Galbanic acid in the root of F. szowitsiana used against promastigotes Leishmania major were concluded to be effective (19).The effectiveness of the Galbanic acid in the aforementioned studies is one of reasons for choosing these plants in the present study. This study aimed to examine the effect of $F$. szowitsiana and F. rigidula extracts on the growth and proliferation of Trichomonas vaginalis in vitro. The study of Azadbakhat et al. which was on the effect of sagebrush juniper essential oil and thyme on the growth of Trichomonas vaginalis showed their antiTrichomonas effect 1-4 hours after adding to the medium culture (24). Rezayian and Colleagues studied the effect of Allium hirtifolium with Metronidazole and it was found that low concentrations of the extract stopped the growth of Trichomonas in the short period (25). The results of the study of Azadbakht et al. on the effect of the liquid extract derived from bulbs of three species of Allium on the giardia cysts revealed the cytotoxic effect of the extracts at different concentrations ranging from 
30 to $90 \%$ (26). The results of recent research were in consistent with the aforementioned studies and Indicated that Allium had cytotoxic effects over different pathogens such as Trichomonas. Moon et al. studied the effect of the concentrations, 1 and $0.5,0.1 \%$, of two types of lavender essential oil (lavandula angustifolia, lavandula intermedia) on Trichomonas vaginalis and found out that the concentration of $0.1 \%$ lavender essential oil wiped out all parasites in 55 minutes (27). Azadbakht and Colleagues study aiming to evaluate the effect of thyme essential oil on Trichomonas vaginalis found that thyme essential oil at the concentration of $0.1,0.01,0.001,0.004$ in the beginning of culture with concentrations of 0.0002 and $0.0001 \%$ up to an hour after the cultivation led to destruction of Trichomonas parasites (26). Mountain tea or shepherd's tea used to treat infections of women in Chaharmahal and Bakhtiari province. The conducted studies examined in the antinociceptive, anti-anxiety and antimicrobial effects of this plant. In Sereshti and Colleagues study, ethanol and aqueous extracts of these plants were not effective in the treatment of trichomoniasis; however, eucalyptus had a very strong anti Trichomonas effect (28). Kalzada and Colleagues, in their in vitro study on examining the antibacterial activity of Trichomonas in Mexican medicinal plants found out that thyme with a minimum inhibitory concentration of $126.4 \mu \mathrm{g} / \mathrm{ml}$ has been inactive for inhibiting trophozoite Trichomonas (29). Muelas and Colleagues studied the effects of the extracts of Mikania cordifolia from Asteraceae family, Scutia buafulia from Asteraceae family and Lobalia neurolarea from Rhamnaceae family and realized that they had the greatest effect on Trichomonas vaginalis (30). Klinghardt and Colleagues have pointed to the role of Propolis in inhibiting protozoa diseases such as trichomoniasis, amoebiasis, toxoplasmosis and giardiasis (31). Staryzyk and Colleagues observed the cytotoxicity of Propolis extract on Trichomonas vaginalis they also showed the extract can kill Toxoplasma gondii after a 24-hour contact (32). The results of Ezatpour's study entitled the effect of essential oil of lavender on Trichomonas vaginalis in vitro showed that while the parasites remained alive for 48 hours in CPLM medium, they were disappeared in the vicinity of Metronidazole after two hours and in DMSO medium after 5 hours. The concentration of 0.1 of lavender essential oil wiped out all live parasites in 90 minutes whereas it lasted120 min and six hours for the concentrations of 0.1 and 0.001 respectively. This study showed that Lavender essential oil had a significant effect on Trichomonas vaginalis (6). The results of Ezatpour and Colleagues, study entitled the effect of essential oil of Artemisia mountain and thyme on Trichomonas vaginalis showed that Trichomonas parasite survived 72 hours in culture medium, but disappeared in the vicinity of Metronidazole and DMSO medium after 1 and 6 hours respectively. Therefore, given the significant impact of these plants, the effect of a separate extract, combination of three plant extracts, on parasite must be studied in vitro (7). Jonkov et al. reported that
Lavandula angustifolia and Mentha piperita essential oils are among the strong Trichomoniasis (8). However, the results of Teresa et al. study on the comparison of the antiTrichomonas effect of gossypol and Metronidazole in vitro showed that the effect of Metronidazole on Trichomonas with same concentration was ten times stronger than gossypol (9). Hosseinzadeh and Colleagues, in a study to evaluate the anti-inflammatory activity of Thymus vulgaris (Lamiaceae) extract in mice and rats concluded that thyme extract showed significant anti-inflammatory activity against acute and chronic inflammation (10). Jafari and Colleagues in their study entitled anti-inflammatory effect of thyme found that this plant has significant antiinflammatory effect which is related to its essential oils and flavonoids (7). Simbar and Colleagues study conducted on the effect of Thymus vulgaris vaginal cream for bacterial vaginosis indicated that $18.6 \%$ of patients experienced complications following the use of Thymus vulgaris vaginal cream and cream acceptance was 96\% (11).

\section{Conclusion}

The results suggested that all examined species had antiTrichomonas activity 24 hours after incubation. However, based on the executive protocol in this study, it was determined that after 72 hours of incubation, the methanol extract of $F$. szowitsiana with 0.360 dose $\mathrm{mg} / \mathrm{ml}$ led to the mortality of $50 \%$ Trichomonas vaginalis trophozoite. Using 2 and $3 \mathrm{mg} / \mathrm{ml}$ doses of methanol extract of $F$. szowitsiana caused the death of all parasites and inhibition of their proliferation. Although chromatography was not done in this study, we used the compounds in the roots of these plants and results were evaluated after 72 hours of incubation through Mack master lam. This study showed that Trichomonas vaginalis was sensitive to this drug fraction. The effectiveness of Galbanic acid in the aforementioned studies was one of reasons for choosing these plants in the present research. In sum, considering the results of this study and the studies of Iranshahi , Fazli bazaz and Colleagues, it seems that Galbanic acid is one of the active compounds in extract of these plants roots and therefore, it can also be the cause anti-protozoan in this plant. However, further studies are needed to prove it. Also based on the statistical analysis, there was a significant difference between different concentrations and inhibition of trophozoite so that the inhibition of trophozoite also increased with increasing of concentration. Therefore, it is hoped that with further studies and identification of effective Allium compounds in the examined species and reviewing their impact in vivo, an appropriate drug can be found for the treatment or recovery of the lesions resulting from trichomoniasis in patients.

\section{Ethical issues}

The local ethics committee approved the study.

\section{Conflict of interests}

Authors declare that there is no any conflict of interests. 


\section{Acknowledgments}

This study was approved and funded by Islamic Azad University, Marand Branch. We would also like to render our thanks to Ms. Shahrbanoo naderi for her cooperation and Dr. Esmail fallah for his erudite guidance.

\section{References}

1. Markell E, Voge M, John D. Medical Parasitology. 7th ed. Philadelphia: W.B. Saunders, 1992

2. Bekhradi R. A new treatment plant. 1th ed. Tehran: Tehran University; 1993. pp. 23-8. (In Persian)

3. Mozaffarian V. Flora of Iran umbelliferus. No. 54. Research Institute of Forests and Rangelands, Tehran, pp. 406-378, 1386. (In Persian)

4. Heymann DL. The world health report 2007: a safer future: global public health security in the 21 st century. Geneva:WHO;2007.

5. Iranshahi M. Purification and structure determination of compounds in the root coma. J Med Plants 2010; 9 (33):73-77. (In Persian)

6. Ezatpur B, Badparva E, Ahmadi S, Rashidipur M, Ziaiye $\mathrm{H}$. Investigation of anti trichomonas vaginalis activity of lavandula angyustifolia essential oil in in vitro media. J Ilam Univ Med Sci 2009; 16(4): 31-6. (In Persian)

7. Jaffari F, Ghannadi AR, Siahpoush A. Antiinflammatory activity of Zataria Multiflora Boiss. J Esfahan Univ Med Sci 2000; 5(2):6-9. (In Persian)

8. Jankov N, Baltova E, Topalov V. Action of some essential oils on Trichomonas vaginalis. Folia Med (Plovdiv) 1968; 10(5):308-10.

9. Teresa MG, Garza M, Said-Fernandez S. In- vitro anti-trichomonal effectiveness of a Gossy-pol metronidazol Blend. Int J Antimicrob Agents 1997; 9: 57-60.

10. Hosseinzadeh H, Ramezani Mohammad, Salmani G. Antiniciceptive and anti-inflamatory and acute toxicity effect of Zataria multiflora Boiss Extracts in mice and rats. J Ethnopharmacol 2000; 73(3):379-85.

11. Simbar M, Azarbad M, Mojab F, Majd HA. A comparative study of the therapeutic effect of the Zataria multiflora vaginal cream and Metronidazol vaginal gel on bacterial vaginosis. Phytomedicine 2008; 15:1013-25.

12. Hatem G, Ardehali P, Motazedian M, Sajjadi M, Fakor Ziba M. Methods for isolation and identification of Leishmania parasites. 1th ed. Shiraz; Shiraz University of Medical Sciences;2007. p. 172-9. (In Persian)

13. Atta-ur-Rahman, Choudhary MI, Thomson WJ. Bioassay techniques for drug development. Netherlands: Harwood Academic Publishers;2005.

14. Dutta A, Mandal G, Mandal C, Chatterjee M. In vitro antileishmanial activity of Aloe vera leaf exudate: A potential herbal therapy in leishmaniasis. Glycoconj J 2007; 24:81-6.

15. Emami A, Mahmoudi M, Arba S, Ahi AS. Prevalence of Leishmania species Artemisia plumbing Razavi Khorasan province in vitro. J Kurdistan Univ Med
Sci 2008; 13:15-20. (In Persian)

16. Shahverdi AR, Fakhimi A, Zarrini G, Dehghan G, Iranshahi M. Galbanic Acid from Ferula szowitsiana enhanced the antibacterial activity of penicillin $G$ and Cephalexin against Staphylococcus aureus. Biol Pharm Bull 2007; 30(9) 1805-7.

17. Fazly Bazzaza BS, Rezaei Du A, Iranshahi M, Naderinasab M, Khajeh Karamodin M. Evaluating the Potentiating Effect of Galbanic acid from Ferula szowitsiana on three common antibiotics against resistant hospital isolates of Staphylococcus aureus. Iran J Pharm Res 2009; 8(3):217-221.

18. Fazly Bazzaz BS, Iranshahi $M$, Naderinasab $M$, Hajian S, Sabeti Z, Masumi E. Evaluation of the effects of galbanic acid from Ferula szowitsiana and conferol from F. badrakema, as modulators of multidrug resistance in clinical isolates of Escherichia coli and Staphylococcus aureus. Res Pharm Sci 2010; 5(1): 25-32.

19. Iranshahi $M$, Arfa $P$, Ramezani $M$, Jaafari $M R$, Sadeghian H, Bassarello C, et al. Sesquiterpene coumarins from Ferula szowitsiana and in vitro antileishmanial activity of 7-prenyloxycoumarins against promastigotes. Phytochemistry 2006;68: 554-61.

20. Sahebkar A, Iranshahi M. Biological activities of essential oils from the genus Ferula (Apiaceae). Asian Biomedicine 2010;4:835-847.

21. Appendino G, Mercalli E, Fuzzati N, Arnoldi L, Stavri $\mathrm{M}$, Gibbons S, et al. Antimycobacterial coumarins from the Sardinian giant fennel (Ferula communis). J Nat Prod 2004;67(12):2108-10.

22. Barthomeuf $C$, Lim S, Iranshahi $M$, Chollet $P$. Umbelliprenin from Ferula szowitsiana inhibits the growth of human M4Beu metastatic pigmented melanoma cells through cell-cycle arrest in G1 and induction of caspase-dependent apoptosis. Phytomedicine 2008; 15:103-11.

23. Cudmore SL, Delgaty KL, Hayward-McClelland SF, Petrin DP, Garber GE. Treatment of infections caused by metronidazole-resistant Trichomonas vaginalis. Clin Microbiol Rev 2004; 17:783-93.

24. Petrin D, Delgaty K, Bhatt R, Garber G. Clinical and microbiological aspects of Trichomonas vaginalis. Clin Microbiol Rev 1998; 11:300-317.

25. Rezaeian M, Taran M, Izaddoost $M$. In vitro Antitrichomonas Activity of Allium Hirtifloium (Persian Shallot) in Comparison with Metronidazole. Iran J Public Health 2006; 35(1):92-94. (In Persian)

26. Azadbakht M, Ziai H, Abdollahi F, Shabankhani B. Effect of essential oils of Artemisia Aucheri Bioss., Zataria Multiflora Boiss. and Myrtus Communis L. on Trichomonas Vaginalis. Quart J herb Med 2006; 8:35-40. (In Persian)

27. Moon T, Wilkinson JM, Cavanagh HM. Antiparasitic activity of two Lavandula essential oils against Giardia duodenalis, Trichomonas vaginalis and Hexamita inflate. Parasitol Res 2006; 99(6):722-728. 
28. Youse HA, Kazemian A, Sereshti M, Rahmanikhoh E, Ahmadinia E, Rafaian M, et al. Effect of Echinophora platyloba, Stachys lavandulifolia, and Eucalyptus camaldulensis plants on Trichomonas vaginalis growth in vitro. Adv Biomed Res 2012; 1: 79.

29. Calzada F, Yepez L, Tapia A. Effect of mexician medicinal plant used treat trichomoniasis on Trichomonas vaginalis trophozoites. J Ethnopharmacol 2007; 113(2):248-251.

30. Muelas-Serrano S, Nogal JJ, Martinez-Diaz RA, Escario JA, Martinez-Fernandez AR, Gomez-Barrio
A. In vitro screening of American plant extracts on Trypanosoma cruzi and trichomonas vaginalis. J Ethnopharmacol 2000; 71: 101-7.

31. Klinghardt DK. Lyme disease: a look beyond antibiotics. Explor Infect Dis 2005; 14: 6-11.

32. Starzyk J, Scheller S, Szaflarski J, Moskwa M, Stojko A. Biological properties and clinical application of propolis. II. Studies on the antiprotozoan activity of ethanol extract of propolis. Arzneimittelforschung 1977; 27(6): 1198-9.

Copyright ( 2014 The Author(s); This is an open-access article distributed under the terms of the Creative Commons Attribution License (http://creativecommons.org/licenses/by/4.0), which permits unrestricted use, distribution, and reproduction in any medium, provided the original work is properly cited. 